\title{
Hyperoxia induces endoplasmic reticulum stress-associated apoptosis via the IRE1 $\alpha$ pathway in rats with bronchopulmonary dysplasia
}

\author{
XIN TONG, MENGYUN LI, NA LIU, WANJIE HUANG, XINDONG XUE and JIANHUA FU \\ Department of Pediatrics, Shengjing Hospital of China Medical University, Shenyang, Liaoning 110004, P.R. China
}

Received September 1, 2019; Accepted July 6, 2020

DOI: $10.3892 / \mathrm{mmr} .2020 .11671$

\begin{abstract}
Bronchopulmonary dysplasia (BPD) is the most common chronic lung disease in premature infants, and alveolar dysplasia and pulmonary vascular development disorders are the predominant pathological features. Apoptosis of lung epithelial cells is a key factor in the pathological process of alveolar developmental arrest. Endoplasmic reticulum stress (ERS)-associated apoptosis is a noncanonical apoptotic pathway involved in the development of several pulmonary diseases.Previous studies have demonstrated that protein kinase RNA-like endoplasmic reticulum kinase, inositol-requiring enzyme $1 \alpha$ (IRE1 $\alpha)$ and activating transcription factor 6 can initiate the apoptosis signaling pathway mediated by ERS and induce apoptosis of injured cells. Among them, the IRE1 $\alpha$ pathway is the most conservative pathway in the unfolded protein response, which serves an important role in a number of pathological environments, to the extent of determining cell fate; however, it is rarely reported in BPD. Based on the establishment of a rat BPD model, the present study verified the activation of ERS in BPD and further confirmed that prolonged ERS inhibited the protective pathway, IRE1 $\alpha / \mathrm{X}$-box binding proteins, and activated the proapoptotic pathway, IRE1 $\alpha / \mathrm{c}-$ Jun N-terminal kinase, to induce the apoptosis of lung epitheliums.
\end{abstract}

\section{Introduction}

Bronchopulmonary dysplasia (BPD) is the most common chronic lung disease in premature infants worldwide, and the predominant pathological features are alveolar dysplasia and pulmonary vascular development disorders $(1,2)$. In

Correspondence to: Dr Jianhua Fu, Department of Pediatrics, Shengjing Hospital of China Medical University, 36 Sanhao Street, Heping, Shenyang, Liaoning 110004, P.R. China

E-mail: slbbest@163.com

Key words: bronchopulmonary dysplasia, apoptosis, endoplasmic reticulum stress, serine/threonine-protein kinase/endoribonuclease inositol-requiring enzyme $1 \alpha, \mathrm{X}$-box binding protein 1 , c-Jun $\mathrm{N}$-terminal kinase, lung total, 10,000 infants are diagnosed with BPD annually in the United States (3). Infants with BPD require respiratory support in early life and for long-term pulmonary dysfunction, including persistent airway obstruction and distal lung development $(1,4)$. Furthermore, infants with BPD often show delays in the development of the nervous system (5). Given that there are no effective preventive and therapeutic measures, BPD remains one of the most challenging issues in neonatology.

A previous study suggested that premature lung exposure to hyperoxia induces lung epithelial cell damage, which triggers pathological changes in alveolar dysplasia (6). In addition, the apoptosis of lung epithelial cells is a key factor in the pathological process of alveolar developmental arrest (7). The two main apoptosis pathways are the death receptor pathway and the mitochondrial pathway $(8,9)$. Previous studies have demonstrated that endoplasmic reticulum stress (ERS)-related apoptosis is a noncanonical apoptosis pathway involved in several lung diseases, including chronic obstructive pulmonary disease (COPD), interstitial lung disease and lung cancer (10-13). The ERS-mediated apoptosis pathway has been investigated; however, only a few studies have assessed its role in BPD (14). A previous study identified that the ER is significantly enlarged in alveolar type II epithelial (AEII) cells of BPD rats (15), suggesting that functional changes in the ER may be involved in the development of BPD; however, the specific molecular mechanism requires further investigation.

Previous studies have demonstrated that protein kinase RNA-like endoplasmic reticulum kinase (PERK), inositol-requiring enzyme $1 \alpha$ (IRE1 $\alpha)$ and activating transcription factor 6 (ATF6) can initiate the ERS-mediated apoptosis signaling pathway, and thereby induce apoptosis in injured cells $(16,17)$. The IRE1 $\alpha$ pathway, which is the most conserved and important pathway in the unfolded protein response (UPR), serves a key role within several pathological processes and determines cell fate (18-21). For example, an increasing amount of evidence has demonstrated that IRE1 $\alpha$ plays a key role in the initiation, growth, metastasis and angiogenesis of cancer cells. The X-box binding protein (XBP1) branch of IRE1 provides tumor cells with the ability to adapt to unfavorable microenvironmental conditions and promote their survival and growth (18). In addition, IRE1 is also involved in important physiological processes, such as lipid metabolism, cell differentiation, inflammation and energy regulation. IRE1 $\alpha$-knockdown 3T3L1 cells were found to have 
notable defects in adipogenesis (19). However, studies on the role of the IRE1 $\alpha$ pathway in BPD are limited.

Thus, the present study established a rat BPD model to verify the presence of ERS activation in BPD, and to determine the role of the IRE1 $\alpha / \mathrm{X}$-box binding proteins (XBP1s)/c-Jun N-terminal kinase (JNK) signaling pathway components: IRE1 $\alpha, \mathrm{p}-\mathrm{IRE} 1 \alpha, \mathrm{XBP} 1 \mathrm{~s}, \mathrm{JNK}$ and p-JNK. Changes in gene and protein expression, and the occurrence of apoptosis were monitored to determine the role of the ERS signaling pathway in the pathogenesis of BPD and to provide an experimental basis for the clinical prevention and treatment of BPD.

\section{Materials and methods}

Animal models. A total of 40 Sprague-Dawley (SD) rats (32 females and 8 males, aged 4 weeks, 220-250 g) were purchased from the Experimental Animal Center of China Medical University and all animal experiments were approved by the Animal Ethics committee of China Medical University (Shenyang, China; approval no. 2019PS308K). The rats were housed in a standard pathogen-free environment (SPF level), at a temperature of $22 \pm 2^{\circ} \mathrm{C}$, and relative humidity $60-70 \%$. The rats were fed ordinary feed and sterilized water. The light/dark cycle was $12 \mathrm{~h}$ light/12 $\mathrm{h}$ dark. The rats were mated at a female/male ratio of 4:1, and gave birth naturally at 22 days of pregnancy. The newborn SD rats (together with the mother rats) were randomly divided into 2 groups within $12 \mathrm{~h}$ after birth, model group $(n=40)$ and control group $(n=40)$. According to the method we established in a previous study (22), the model group newborn rats were placed in a glass oxygen box to maintain the oxygen concentration at $80-85 \%$. Soda lime was used to absorb $\mathrm{CO}_{2}$ in the oxygen box to keep the concentration $<0.5 \%$. The silica gel absorbed water to maintain the oxygen level. The temperature of the box was $25-27^{\circ} \mathrm{C}$, the humidity was $60-70 \%$, and the oxygen concentration was monitored with a digital oxygen meter every day. The control rats inhaled fresh air. All additional conditions and control factors were the same in both groups. The oxygen box was opened for $30 \mathrm{~min}$ every $24 \mathrm{~h}$ to replenish food and water and to change the bedding, and maternal rats in the model group and the control group were exchanged to prevent oxygen poisoning.

Collection of lung samples. A total of 8 pups from each group were randomly selected at 1,3,7 and 14 days after the start of the experiment and anesthetized by sevoflurane inhalation. Lung tissues were removed under aseptic conditions and the right middle lobe was fixed with $4 \%$ paraformaldehyde (PFA; Beyotime Institute of Biotechnology), for $>24 \mathrm{~h}$ at room temperature, for hematoxylin and eosin (H\&E) staining, immunohistochemistry (IHC) and terminal deoxynucleotidyl transferase dUTP nick-end labeling (TUNEL). The remaining lung tissue samples were stored at $-80^{\circ} \mathrm{C}$ for protein and mRNA analysis.

Lung histology and morphometric analysis. Lung tissues were fixed with 4\% PFA for 24-48 $\mathrm{h}$ at room temperature, dehydrated in a graded alcohol series. Then, the tissues were embedded in paraffin at room temperature. Paraffin-embedded tissue samples were cut into $4-\mu \mathrm{m}$-thick sections and conventional dewaxing and rehydration was performed. Morphological changes were observed by $\mathrm{H} \& \mathrm{E}$ staining for $10 \mathrm{~min}$ at room temperature. For each group of animals 6 slices at different time points were randomly selected, and 10 visual fields in each slice were randomly selected to observe pathological changes under the high power lens of a light microscope (Nikon C1 System; Nikon Corporation) at magnification $\mathrm{x} 200$. The radial alveolar count (RAC) and mean alveolar diameter (MAD) are simple and relatively accurate measures of lung development, larger RAC values indicate more complete alveolar development and larger MAD values indicate larger alveoli and less mature alveolar development. The RAC was measured using the Emery and Mithal method $(23,24)$, whereas the MAD was measured using Image-Pro Plus 6.0 software (Media Cybernetics, Inc.).

Transmission electron microscopy (TEM). Fresh lung tissues were sampled within 1-3 min, the size of the sampled tissue was $1 \mathrm{~m}^{3}$, as thin as possible. Immediately after removing the tissue, fresh lung tissues were fixed with $2.5 \%$ glutaraldehyde (Beijing Solarbio Science \& Technology Co., Ltd.) for $2 \mathrm{~h}$ at room temperature, and then with $1 \%$ succinic acid, dehydrated with acetone and placed in epoxy resin prior to being impregnated and embedded with epoxy resin for $72 \mathrm{~h}$. Then, the ultra-thin sections were produced and double-stained with uranyl acetate and lead nitrate for $30 \mathrm{~min}$. The ultrastructural changes in alveolar epithelial type II (AEII) cells and organelles were observed by TEM (H-600; Hitachi, Ltd.).

TUNEL staining. TUNEL staining was performed to detect apoptosis in lung tissue. Tissue sections were routinely dewaxed, rehydrated in an ethanol gradient and then washed with PBS. Following which, 3\% hydrogen peroxide $\left(\mathrm{H}_{2} \mathrm{O}_{2}\right)$ was added and sections were incubated at $37^{\circ} \mathrm{C}$ for $20 \mathrm{~min}$ to eliminate endogenous peroxidase activity. Then, the sections were treated with proteinase $\mathrm{K}$ at $37^{\circ} \mathrm{C}$ for $8 \mathrm{~min}$, and washed with PBS three times for $5 \mathrm{~min}$. The sections were then incubated at $37^{\circ} \mathrm{C}$ for $1 \mathrm{~h}$ in a humidified chamber with $50 \mu \mathrm{l}$ of the TUNEL mixture (Roche Diagnostics) per sample, according to the manufacturer's protocol. Subsequently, sections were then stained with DAPI (Beyotime Institute of Biotechnology) at room temperature for $5 \mathrm{~min}$ for nuclear staining. For each group of animals six slices were randomly selected to observe the TUNEL-positive cells under the high power lens of a light microscope (Nikon C1 System; Nikon Corporation) at magnification $\mathrm{x} 200$. The percentage of apoptotic cells was determined as the percentage of the total cells positive for TUNEL.

$I H C$. The paraffin-embedded lung tissue sections were baked in an oven at $60^{\circ} \mathrm{C}$ for $30 \mathrm{~min}$. After routine dewaxing and gradient alcohol rehydration at room temperature, they were thoroughly washed with PBS. Then, $3 \% \mathrm{H}_{2} \mathrm{O}_{2}$ was added and sections were incubated at $37^{\circ} \mathrm{C}$ for $20 \mathrm{~min}$ to eliminate endogenous peroxidase activity. The sections were subjected to heat-mediated antigen retrieval for $8 \mathrm{~min}$ using citric acid, and then cooled to room temperature, and washed with PBS. Goat serum (50 $\mu \mathrm{l}$; OriGene Technologies, Inc.) was used for blocking at $37^{\circ} \mathrm{C}$ for $30 \mathrm{~min}$, and then sections were incubated with primary antibodies against: Glucose regulated protein 78,000 (GRP78; 1:5,000; cat. no. ab21685) phosphorylated (p)-IRE1 $\alpha$ (1:500; cat. no. ab48187) and p-JNK (1:300; 
Table I. Primer sequences used for quantitative PCR.

\begin{tabular}{lll}
\hline Gene & \multicolumn{1}{c}{ Forward $\left(5^{\prime}-3^{\prime}\right)$} & \multicolumn{1}{c}{ Reverse $\left(5^{\prime}-3^{\prime}\right)$} \\
\hline$G R P 78$ & CCGTAACAATCAAGGTCTACGA & AAGGTGACTTCAATCTGGGGTA \\
$X B P 1-s$ & GCACCTCTAAGCTCTTCA & CCTCATATCCACAGTCACT
\end{tabular}

GRP78, glucose regulated protein 78,000; XBP1s, X-box binding protein 1.

cat. no. ab124956; all from Abcam) overnight at $4^{\circ} \mathrm{C}$. Following this, sections were washed four times with PBS, and then incubated with goat anti-mouse/anti-rabbit IgG and horseradish peroxidase-labeled streptavidin (cat. no. SP-9001; OriGene Technologies, Inc.) at $37^{\circ} \mathrm{C}$ for $20 \mathrm{~min}$. Subsequently, DAB (OriGene Technologies, Inc.) development was performed under an optical microscope (Nikon C1 System; Nikon Corporation), and the color development was stopped when brown particles appeared in the nucleus or cytoplasm. For the negative control, the primary antibodies (GRP78, p-IRE1 $\alpha$ and $\mathrm{p}-\mathrm{JNK}$ ) were replaced with PBS; all other steps were performed as aforementioned.

Western blot analysis. Total protein was extracted from lung tissues using RIP (Beyotime Institute of Biotechnology), quantified using a BCA protein assay kit (Beyotime Institute of Biotechnology), and then diluted with loading buffer and boiled for $5 \mathrm{~min}$. Protein samples (50 $\mu \mathrm{g} / \mathrm{lane}$ ) were separated by 10 or $12 \%$ SDS-PAGE and subsequently transferred onto a PVDF membrane (EMD Millipore) at $100 \mathrm{~V}$ for 30 min. Subsequently, the PVDF membrane was blocked with non-fat milk diluted with 5\% TBST at room temperature for $1 \mathrm{~h}$. Membranes were incubated with primary antibodies against: GRP78 (1:2,000; cat. no. ab21685; Abcam), p-IRE1 $\alpha$ (1:2,000; cat. no. ab48187; Abcam), IRE1 $\alpha$ (1:1,000; cat. no. 3294; Cell Signaling Technology, Inc.), XBP1-s (1:1,000; cat. no. 27901S; Cell Signaling Technology, Inc.), p-JNK (1:2,000; cat. no. ab124956; Abcam), JNK $(1: 2,000$; cat. no. ab179461; Abcam) and $\beta$-actin $(1: 4,000$; cat. no. 60008-1-Ig; ProteinTech Group, Inc.) overnight at $4^{\circ} \mathrm{C}$. Following the primary incubation, membranes were incubated with horseradish peroxidase-conjugated secondary antibodies (1:5,000; goat anti-mouse and anti-rabbit IgG-HRP; cat. nos. SA00001-1 and SA00001-2, respectively; Wuhan Sanying Biotechnology) at room temperature for 2 h. Proteins bands were detected using the Super ECL Plus (Thermo Fisher Scientific, Inc.) and luminescence analysis was performed with a chemiluminescence imaging system (C300; Azure Biosystems, Inc.). The bands were standardized according to $\beta$-actin.

Reverse transcription-quantitative $(R T-q) P C R$. RNA was extracted using TRIzol ${ }^{\circledR}$ reagent (Takara Biotechnology Co., Ltd.). According to the manufacturer's protocols, $1 \mathrm{ml}$ TRIzol was added to $50 \mathrm{mg}$ lung tissues and then the tissues were cut into pieces and mixed thoroughly. Chloroform $(200 \mu \mathrm{l})$ was added to the tissues for $5 \mathrm{~min}$ at room temperature, followed by centrifugation $(12,000 \mathrm{x} \mathrm{g})$ at $4^{\circ} \mathrm{C}$ for $15 \mathrm{~min}$ to get the supernatant. Then, isopropanol was added at room temperature for $10 \mathrm{~min}$ and centrifuged $(12,000 \mathrm{x} \mathrm{g})$ at $4^{\circ} \mathrm{C}$ for $10 \mathrm{~min}$. Finally, $75 \%$ ethanol was added three times at room temperature for $5 \mathrm{~min}$. The mRNA was reverse transcribed into cDNA using a Prime Script ${ }^{\mathrm{TM}}$ RT reagent kit (Takara Biotechnology Co., Ltd.) to remove genomic DNA for $15 \mathrm{~min}$ at room temperature, with the following conditions: $37^{\circ} \mathrm{C}$ for $15 \mathrm{~min}$, and $85^{\circ} \mathrm{C}$ for $5 \mathrm{sec}$. The primers (Table I) were designed and synthesized by Sangon Biotech Co., Ltd., and qPCR was performed using a 7500 Real-Time PCR System (Applied Biosystems, Thermo Fisher Scientific, Inc.) and a PCR amplification kit (Takara Biotechnology Co., Ltd.) in a reaction volume of $20 \mu \mathrm{l}$. The thermocycling conditions were as follows: $95^{\circ} \mathrm{C}$ for $30 \mathrm{sec}, 95^{\circ} \mathrm{C}$ for $5 \mathrm{sec}, 60^{\circ} \mathrm{C}$ for $34 \mathrm{sec}$, $95^{\circ} \mathrm{C}$ for $15 \mathrm{sec}, 60^{\circ} \mathrm{C}$ for $1 \mathrm{~min}, 95^{\circ} \mathrm{C}$ for $30 \mathrm{sec}$, and $60^{\circ} \mathrm{C}$ for $15 \mathrm{sec}$, for a total of 40 cycles. Relative expression levels were calculated using the $2^{-\Delta \Delta \mathrm{Cq}}$ method and normalized to $\beta$-actin (25).

Statistical analysis. Statistical analysis was performed using SPSS 17.0 software (SPSS, Inc.). Unpaired Student's t-test was used to compare difference between two groups. $\mathrm{P}<0.05$ was considered to indicate a statistically significant difference.

\section{Results}

Morphological changes in lung tissue and differences in lung development. The H\&E staining results demonstrated that lung tissues in the control group gradually improved with the age of the rats; the alveolar number gradually increased, the alveolar interval became thinner and the irregular alveolar morphology gradually disappeared as the alveoli adopted a uniform size. At day 14, alveolarization was complete. Alveolar development in the model group was significantly delayed compared with the control group, with a significant reduction in the number of alveoli, an increased alveolar diameter, an irregular alveolar shape, simple alveolar structure and unclear alveolar ridges and stimulation intervals (Fig. 1A).

Compared with the control group, the RAC in the model group was significantly decreased starting at day $7(\mathrm{P}<0.05)$ and this difference was more pronounced at day $14(\mathrm{P}<0.01)$. These findings suggested that the model group experienced a block in alveolar development starting at day 7 and development was significantly delayed compared with the control group. Another indicator of alveolar development is MAD, which was significantly greater in the model group compared with the control group at day $7(\mathrm{P}<0.05)$, with a more pronounced increase at day 14 , indicating that the alveoli in the model group gradually became larger compared with those in the control group $(\mathrm{P}<0.01$; Fig. $1 \mathrm{~B}$ and $\mathrm{C})$. 


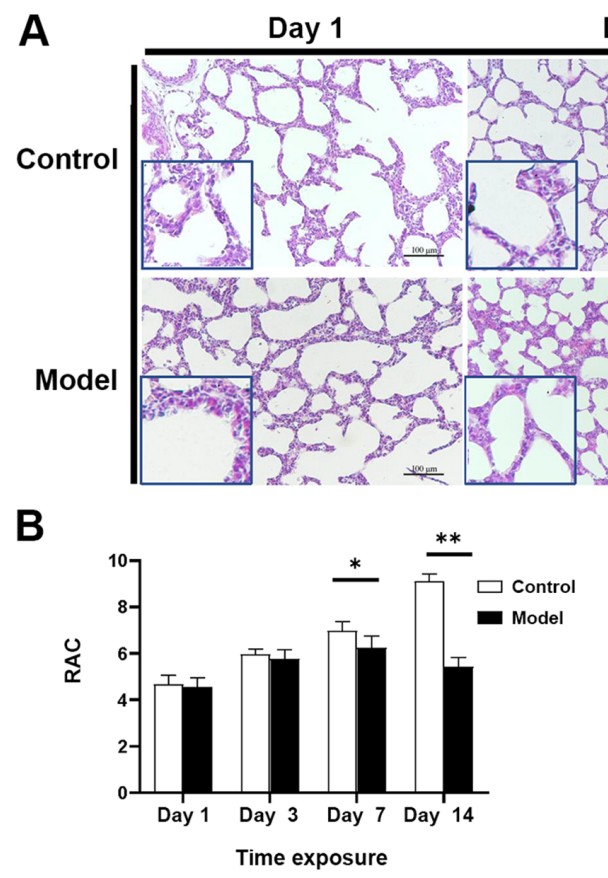
Day 3
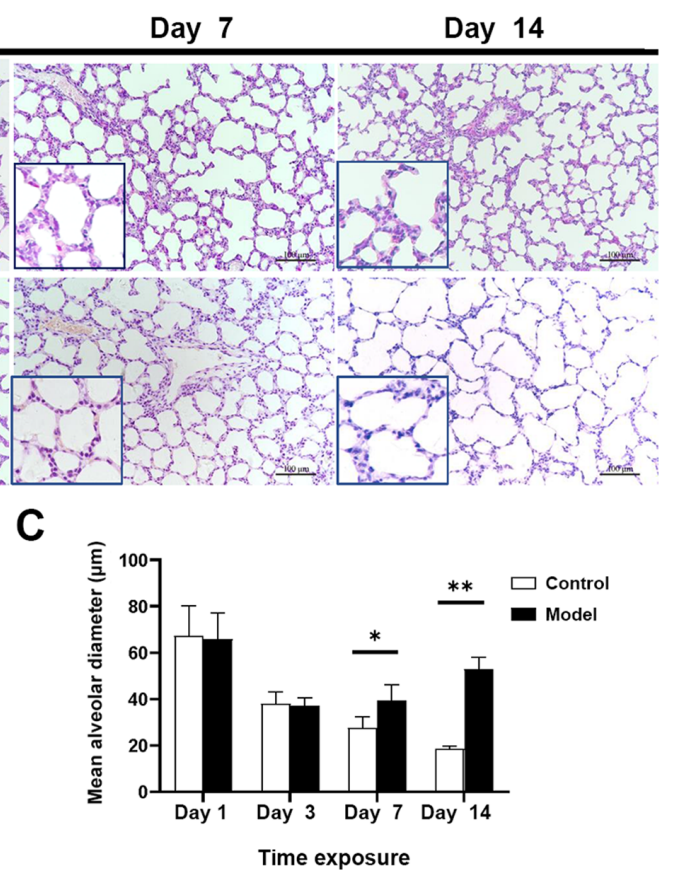

Figure 1. Morphological changes in lung tissue from control and model rats. (A) Hematoxylin and eosin staining (scale bar, $100 \mu \mathrm{m}$ ). (B) The RAC was significantly lower in the model group compared with the control group at day 7, with a greater difference at day 14. (C) The MAD was significantly increased at day 7 in the model group compared with the control group and this difference was more pronounced at day $14 .{ }^{*} \mathrm{P}<0.05,{ }^{* *} \mathrm{P}<0.01$. RAC, radial alveolar count; MAD, mean alveolar diameter.

Ultrastructural changes in AEII cells in lung tissue. TEM demonstrated that the AEII cells in the control group were cubic in form, with microvilli at the top and characteristic lamellar bodies in the cytoplasm. The organelles, including the ER and mitochondria, were normal. In the AEII cells in the model group, chromatin accumulation at the nuclear periphery was observed and the microvilli at the top of the cell were sparse and detached. The cytoplasmic lamellar bodies were destroyed and vacuolization, mitochondrial swelling, ER expansion and degranulation were observed (Fig. 2).

Detection of apoptosis in lung tissue. TUNEL-positive cells were observed in lung tissue at day 14 in the control group, while the number of apoptotic cells in the model group was significantly increased $(\mathrm{P}<0.01$; Fig. 3$)$.

RT-qPCR analysis of GRP78 and XBPI-s mRNA levels in lung tissue. RT-qPCR analysis demonstrated that GRP78 mRNA levels were upregulated by exposure to hyperoxia (Fig. 4A). Compared with the control group, the model group exhibited GRP78 upregulation at day 3, with a significant increase on day $14(\mathrm{P}<0.05)$; GRP78 protein levels demonstrated a similar change (Fig. 5A). Consistent with this finding, XBP1-s mRNA expression peaked on day $3(\mathrm{P}<0.05$; Fig. $4 \mathrm{~B})$ and then began to decline prior to reaching the lowest level on day 14 .

Western blot analysis of GRP78, p-IRE1 $\alpha$, IRE1 $\alpha, X B P 1-s$, p-JNK and JNK protein expression levels in lung tissue. Western blot analysis demonstrated that GRP78 protein expression was upregulated with hyperoxia exposure; GRP78 expression increased beginning on day 3 and was significantly increased on day $14(\mathrm{P}<0.05)$. The $\mathrm{p}-\mathrm{IRE} 1 \alpha / \mathrm{IRE} 1 \alpha$ ratio gradually increased over time; it increased starting at day 3
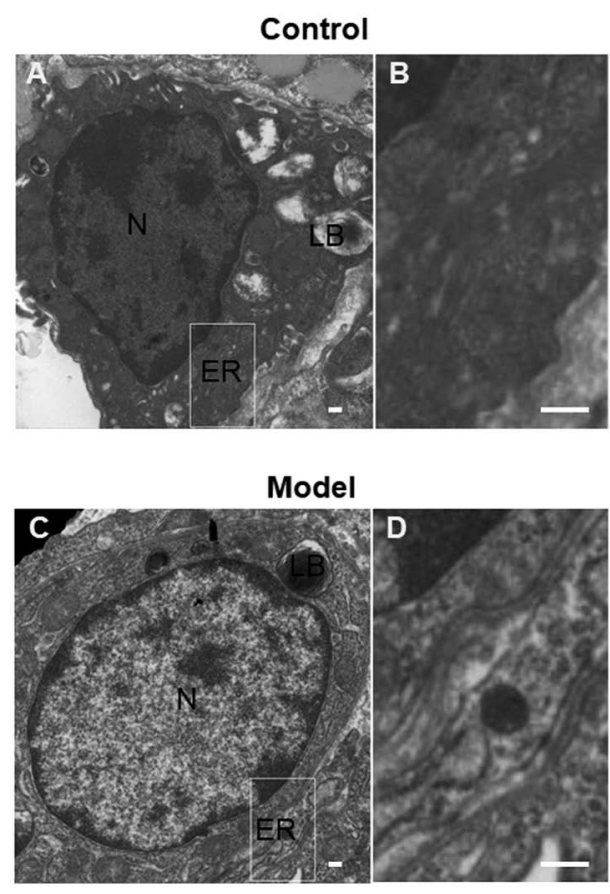

Figure 2. Ultrastructural changes in the ER of AEII cells at day 14 via transmission electron microscopy. (A) Control group at 14 days. (B) Magnification of the insert in (A). (C) Model (hyperoxia) group at 14 days. (D) Magnification of the insert in (C). Compared with control rats, those in the model group demonstrated marked ER dilation. Scale bar, $0.1 \mu \mathrm{m}$. ER, endoplasmic reticulum; N, nucleus; LB, lamellar body.

in the experimental group and peaked at day $14(\mathrm{P}<0.05)$; the expression trend was consistent with that of GRP78. Changes in XBP1-s protein expression were relatively delayed, with the highest expression at day $7(\mathrm{P}<0.05)$ and decreased expression 

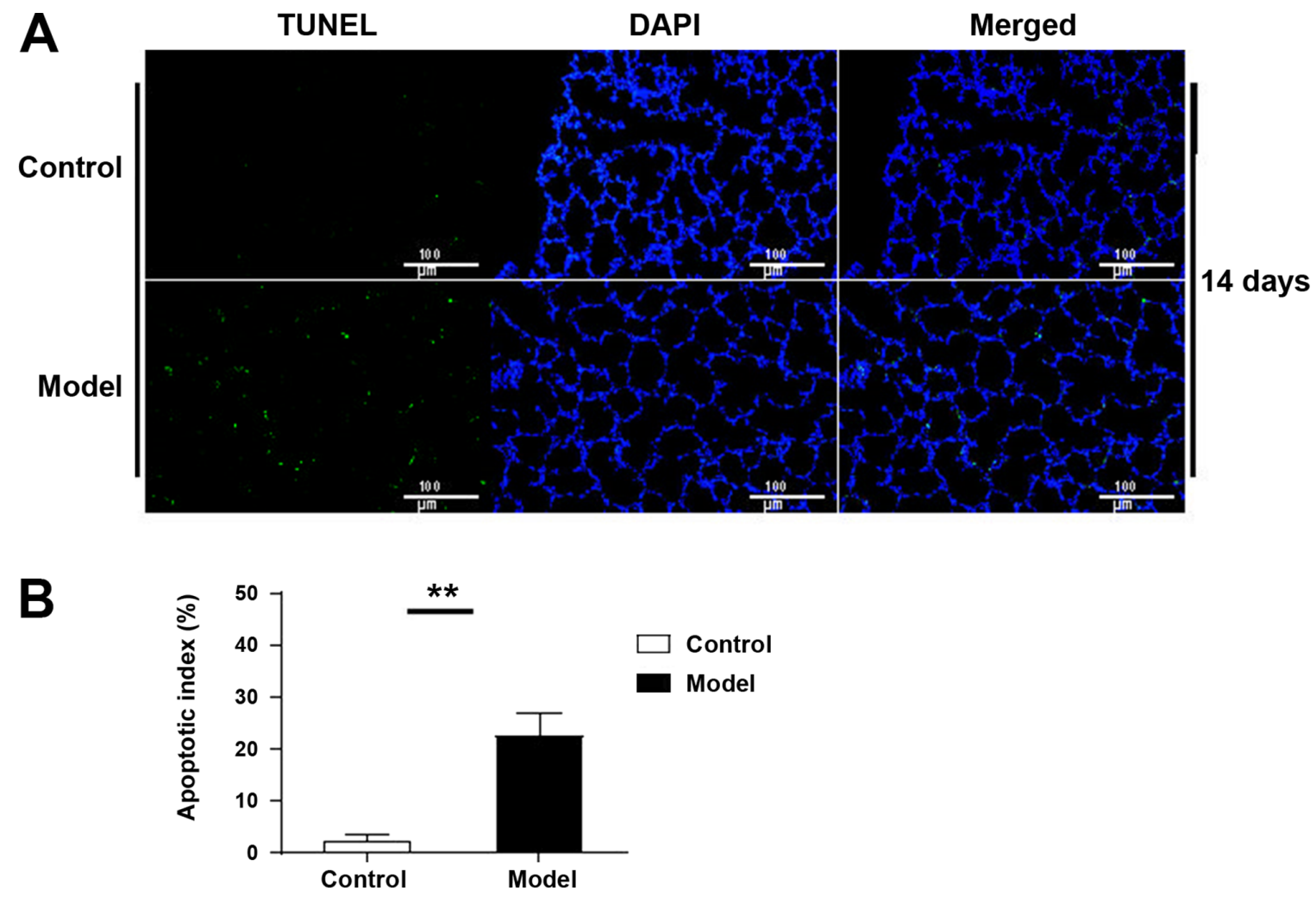

Figure 3. TUNEL fluorescence staining of lung tissues. TUNEL staining demonstrated a significantly increased number of apoptotic cells at day 14 in the model group compared with the control group (scale bar, $100 \mu \mathrm{m}$; magnification, x200). (A) TUNEL fluorescence staining of lung tissues. (B) Apoptotic index. ${ }^{* *} \mathrm{P}<0.01$. TUNEL, terminal deoxynucleotidyl transferase-mediated dUTP nick end labeling.
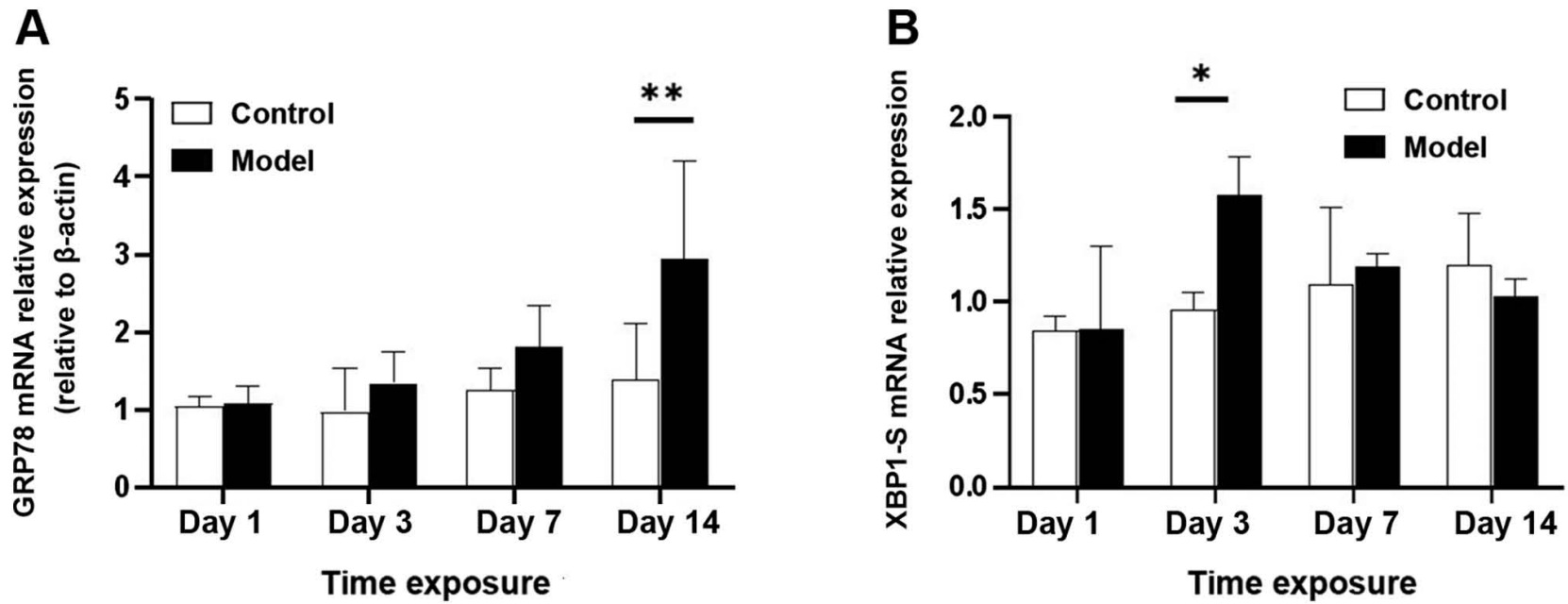

Figure 4. RT-qPCR analysis of GRP78 and XBP1-s mRNA expression in lung tissue. (A) GRP78 mRNA levels gradually increased with hyperoxia exposure and were markedly increased on day 14. (B) XBP1s mRNA expression peaked at day 3 and then began to decrease, reaching the lowest level at day 14 . Relative GRP78/XBP1s mRNA expression was normalized to $\beta$-actin expression and calculated using the $2^{-\Delta \Delta C q}$ method. ${ }^{*} \mathrm{P}<0.05,{ }^{* *} \mathrm{P}<0.01$. GRP78, glucose regulated protein 78,000; XBP1s, X-box binding protein 1.

at day 14 . The $\mathrm{p}-\mathrm{JNK} / \mathrm{JNK}$ ratio was increased on day 7 $(\mathrm{P}<0.01)$ and this increase was more pronounced on day 14 $(\mathrm{P}<0.01$; Fig. 5A-D).

Immunohistochemistry analysis of the localization and expression of GRP78, $p-I R E 1 \alpha, X B P 1$ and $p-J N K$. Immunohistochemistry analysis revealed that in the model group, GRP78 protein was mainly localized in the cytoplasm of AEII cells, closely surrounding the nucleus, consistent with ER localization. p-IRE1 $\alpha$, p-JNK and XBP1 were mainly localized in the nucleus of AEII cells (Fig. 6).

\section{Discussion}

Obstruction of alveolar development is a pathological characteristic in preterm infants with BPD $(1,2)$. In the present study, 
A

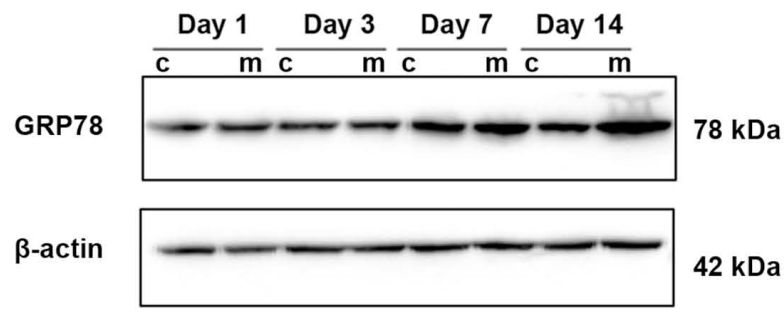

B

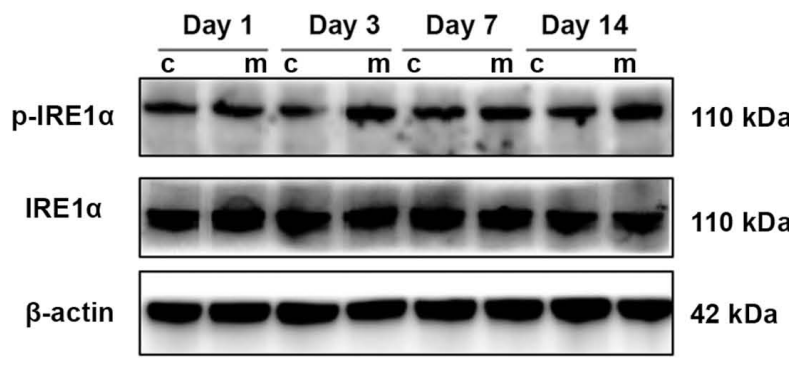

C

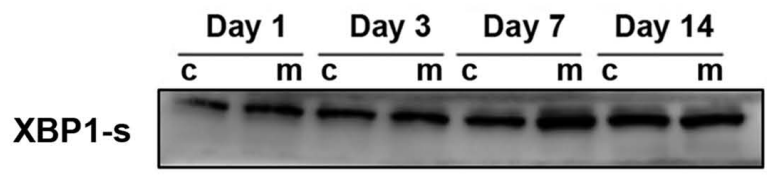

$\beta$-actin

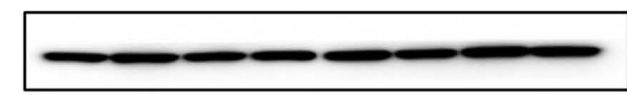

D

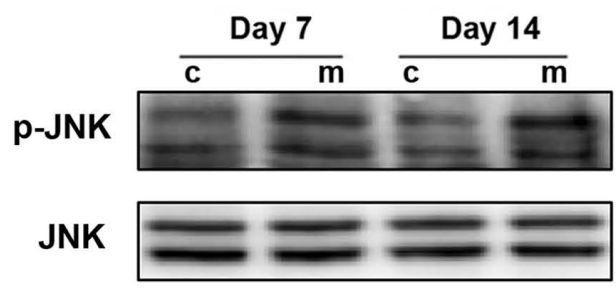

$\beta$-actin

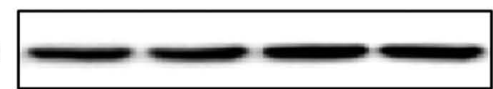

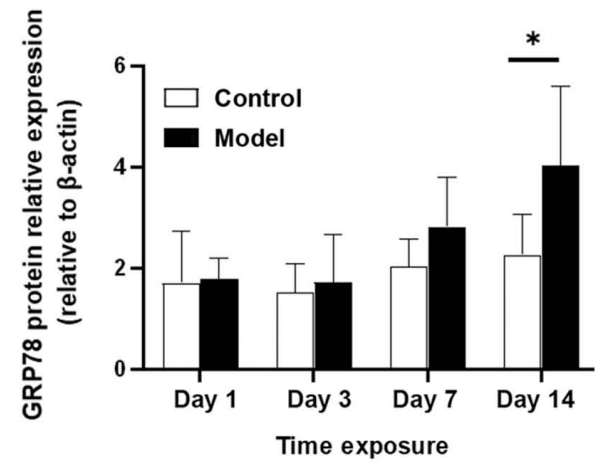
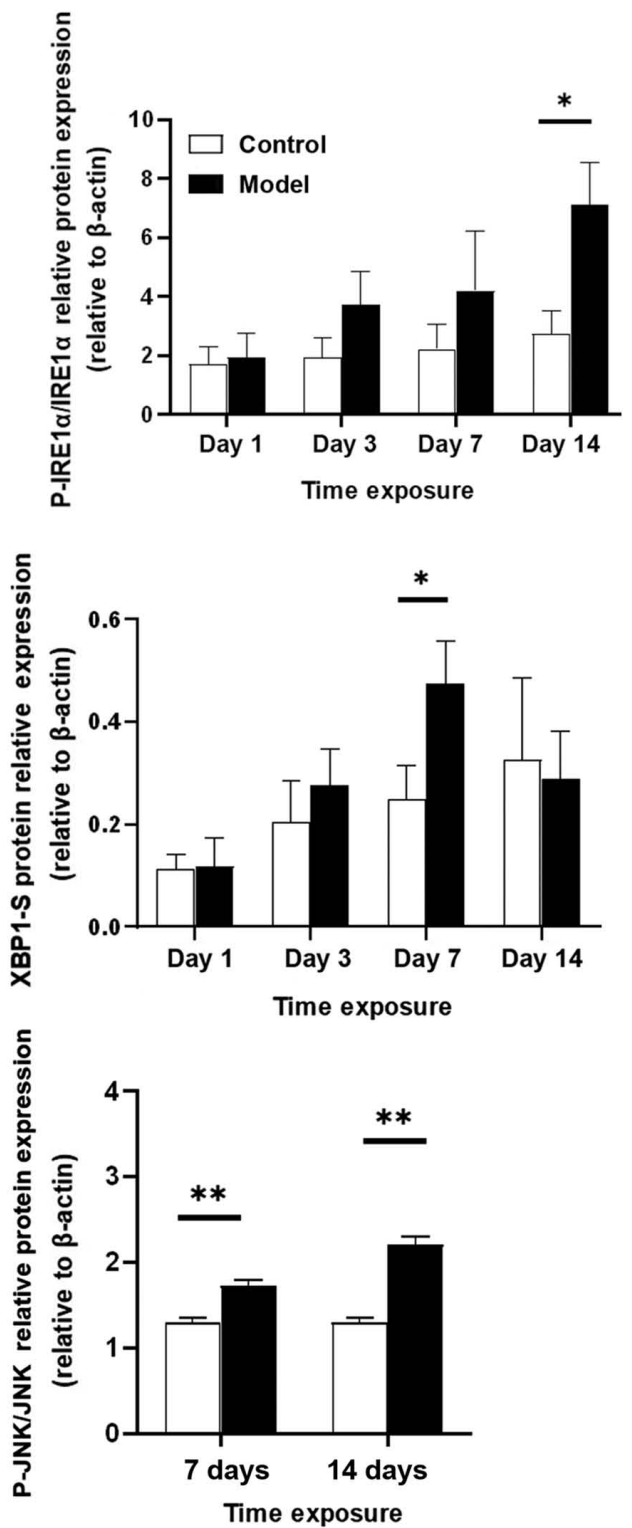

Figure 5. Western blot analysis of GRP78, p-IRE1 $\alpha$, IRE1 $\alpha$, XBP1-s, p-JNK and JNK protein expression levels in lung tissue. (A) GRP78 protein levels gradually increased with hyperoxia exposure and were markedly increased on day 14. (B) Protein levels of p-IRE1 $\alpha$ and IRE1 $\alpha$. The p-IRE1 $\alpha /$ IRE1 $\alpha$ ratio progressively increased over time; it was upregulated in the treatment group beginning at day 3 and was markedly increased at day 14. (C) XBP1s protein expression peaked at day 7 and then began to decrease, reaching the lowest level at day 14. (D) Protein levels of p-JNK and JNK. The p-JNK/JNK ratio increased beginning at day 7 and was significantly increased at day 14 . Relative protein expression was normalized to $\beta$-actin expression. ${ }^{*} \mathrm{P}<0.05,{ }^{* *} \mathrm{P}<0.01$. GRP78, glucose regulated protein 78,000; p-, phosphorylated; IRE1 $\alpha$, inositol requiring kinase enzyme $1 \alpha$; XBP1s, X-box binding protein 1.

upon exposure to hyperoxia, the number of alveoli gradually decreased, the alveoli became larger, the alveolar structure was simplified and alveolar development was blocked. The RAC is an important indicator of the degree of alveolarization (24).
Compared with the control group, the RAC in the model group was significantly decreased at day 7 of hyperoxia exposure and further decreased with increasing age. RAC analysis also demonstrated the stagnation of alveolar development in the 


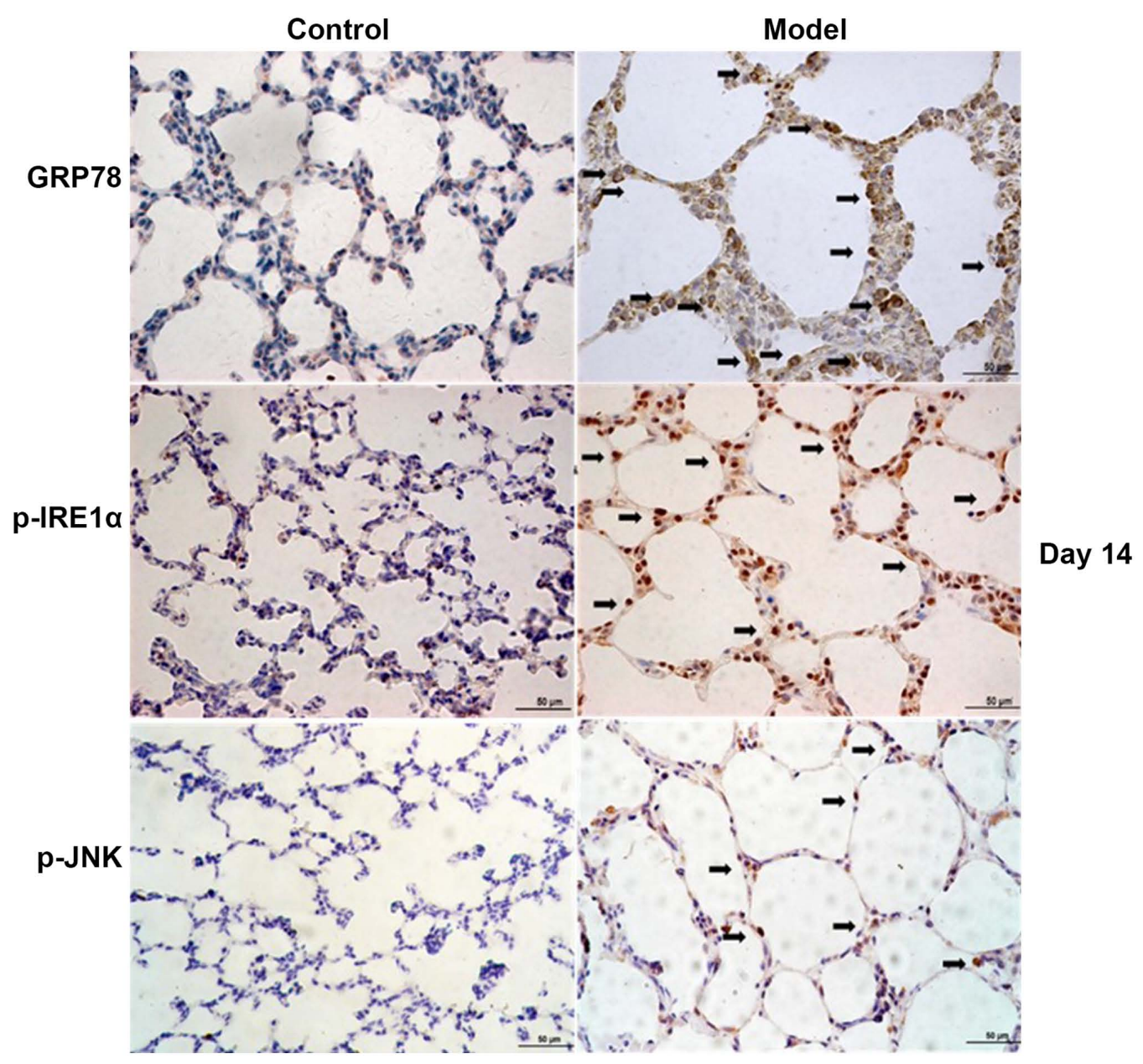

Figure 6. Detection of GRP78, p-IRE1 $\alpha$, XBP1 and p-JNK protein expression in lung tissue from the control and model groups at different time points by immunohistochemistry (magnification, $\mathrm{x} 400$ ). The positively stained cells appeared brown-yellow. GRP78 protein was primarily localized in the cytoplasm of AEII cells. GRP78, glucose regulated protein 78,000; p-, phosphorylated; IRE1 $\alpha$, inositol requiring kinase enzyme $1 \alpha$; XBP1s, X-box binding protein 1; AEII, alveolar type II epithelial.

model group. These pathological changes are consistent with the pathological features of alveolar development in BPD.

When ERS occurs, the UPR survival pathway is initiated. Three UPR signaling pathways are mediated by ATF6, PERK and IRE1, and a series of cascading pathways are activated to increase protein processing capacity and protein folding ability by reducing protein translation. The increased degradation of misfolded proteins relieves the pressure on the ER and promotes cell survival $(16,17)$. Previous studies have demonstrated that ERS serves an important role in lung diseases, including COPD, pulmonary fibrosis, acute lung injury and lung cancer (10-13). GPR78 is significantly upregulated by ERS only and is extensively used as a marker of ERS $(26,27)$. In the present study, the ultrastructure of AEII cells in the lung tissue of the model group demonstrated substantial hyperplasia and expansion, suggesting a dysfunctional ER structure and a significant increase in GRP78 gene and protein expression. Taken together, these results indicated that the activation of ERS was involved in the development of BPD, which was consistent with the reports of Lu et al (14) and Teng et al (28).

Among the three signaling pathways that mediate the UPR, the IRE1 $\alpha$ pathway is the most sensitive and conservative $(29,30)$. IRE1 $\alpha$ is a kinase/endonuclease (RNase) with multiple activities. Upon ERS activation, IRE1 $\alpha$ is immediately activated by oligomerization and autophosphorylation. When activated, the RNase domain of IRE1 $\alpha$ excises the 26-nucleotide intron from the XBP1-u mRNA and cleaves the exon by $R t c B$ ligation to generate the active transcription factor XBP1-s, an important protective factor in cells. Upon activation, XBP1-s immediately enters the nucleus and upregulates its target genes, including those that encode ER chaperone and ER-associated degradation components, thereby enhancing the folding and degradation of proteins and maintaining ER homeostasis. However, with prolonged overactivation of ERS, XBP1-s activation is gradually inhibited and the apoptosis pathway is progressively activated (). Previous studies have demonstrated that when ERS is overactivated, IRE1 $\alpha$ activates JNK by interacting with tumor necrosis factor (TNF) receptor-associated factor 2 (TRAF2) (29-32). Activated JNK can induce apoptosis through the death receptor pathway or the mitochondrial pathway (32).

Previous studies have demonstrated that the protective effects of XBP1 are important in a number of diseases and targeting these protective effects has been proposed as a strategy for the treatment of these diseases (33-37). Akiyama et al (33) reported that XBP1 can alleviate glucose dysfunction by improving insulin sensitivity and stimulating insulin secretion. Furthermore, Casas-Tinto et al (34) identified that the 
spliced form of XBP1 attenuates neuronal damage in cell culture models of Alzheimer's disease. In addition, a previous study demonstrated that XBP1 is critical for the differentiation of dendritic cells (37). These previous studies proposed that targeting these protective effects could be a strategy for the treatment of these diseases.

In the present study, western blotting and immunohistochemistry analyses demonstrated that IRE1 $\alpha$ phosphorylation increased with hyperoxia exposure, which was consistent with the trend in GRP78 levels, indicating that IRE1 $\alpha$ is rapidly activated following ERS activation. XBP1-s mRNA levels peaked on day $3(\mathrm{P}<0.05)$ and then began to decline, reaching the lowest level on day 14. Changes in XBP1-s protein levels were relatively delayed, peaking at day 7 and then declining. These findings indicated that the IRE1 $\alpha / \mathrm{XBP} 1$ pathway is activated at the onset of ERS. However, over time, XBP1-s activation was gradually inhibited when ERS was overactivated. In addition, the changes in XBP1-s mRNA and protein levels in the control group demonstrated that XBP1-s expression increased with increasing age, suggesting that this protein may be associated with lung development. This finding further indicated that excessive ERS led to the inhibition of XBP1-s and may be responsible for the obstruction of alveolar development in BPD.

When ERS was overactivated, in addition to downstream XBP1 inhibition and IRE1 $\alpha / \mathrm{JNK}$ pathway activation, the induction of apoptosis was observed. Previous studies have demonstrated that JNK-induced apoptosis is an important cause of several diseases, including cancer, liver dysfunction, metabolic disorders and neurodegenerative diseases (38-41). The apoptosis of lung epithelial cells is an important mechanism of BPD (1). It has been demonstrated that the apoptosis-related gene, Fas/FasL initiates apoptosis in caspase-dependent AT-II cells and that the signal is transduced by Bcl-2/Bax; these events are key in BPD-related alveolar dysplasia (42). In addition, the present study demonstrated that when BPD developed and the change in RAC became evident,ERS was overactivated, XBP1-s began to decrease and JNK activation gradually increased; these differences were more pronounced at day 14. Furthermore, TUNEL experiments confirmed that apoptosis was significantly increased at day 14. These findings suggest that the apoptosis pathway is activated when ERS is overactivated.

Taken together, the present study demonstrated that ERS was present during the development and progression of BPD, and that ERS overactivation inhibited the protective IRE1 $\alpha /$ XBP1-s pathway and activated the proapoptotic IRE1 $\alpha /$ JNK pathway to induce the apoptosis of lung epithelial cells, which may lead to BPD. The key factors in the pathogenesis of alveolar dysplasia have been elucidated; however, further research is required to clarify the exact mechanism.

\section{Acknowledgements}

The authors would like to thank Dr Liu Dongyan (Laboratory Research Center, Shengjing Hospital of China Medical University, Shenyang, China) for her technical assistance.

\section{Funding}

The present study was supported by the National Natural Science Foundation of china (grant no. 81571479).

\section{Availability of data and materials}

The datasets used and/or analyzed during the present study are available from the corresponding author on reasonable request.

\section{Authors' contributions}

XT designed the study and contributed to manuscript writing. ML collected the rat lung tissue and contributed to establishing the bronchopulmonary dysphasia rat model. NL and WH contributed to analysis and interpretation of data. JF made contributions to the conception and design of the project. XX contributed to the design of the study and revised the work critically for important intellectual content. All authors read and approved the final manuscript.

\section{Ethics approval and consent to participate}

All animal experiments were approved by the Animal Ethics committee of China Medical University (Shenyang, China; approval no. 2019PS308K).

\section{Patient consent for publication}

Not applicable.

\section{Competing interests}

The authors declare that they have no competing interests.

\section{References}

1. Kalikkot Thekkeveedu R, Guaman MC and Shivanna B: Bronchopulmonary dysplasia: A review of pathogenesis and pathophysiology. Respir Med 132: 170-177, 2017.

2. Voynow JA: 'New' bronchopulmonary dysplasia and chronic lung disease. Paediatr Respir Rev 24: 17-18, 2017.

3. Stoll BJ, Hansen NI, Bell EF, Shankaran S, Laptook AR, Walsh MC, Hale EC, Newman NS, Schibler K, Carlo WA, et al: Neonatal outcomes of extremely preterm infants from the NICHD neonatal research network. Pediatrics 126: 443-456, 2010.

4. Hwang JS and Rehan VK: Recent advances in bronchopulmonary dysplasia: Pathophysiology, prevention, and treatment. Lung 196: 129-138, 2018.

5. DeMauro SB: The impact of bronchopulmonary dysplasia on childhood outcomes. Clin Perinatol 45: 439-452, 2018.

6. Bhandari V: Hyperoxia-derived lung damage in preterm infants. Semin Fetal Neonatal Med 15: 223-229, 2010.

7. Pagano A and Barazzone-Argiroffo C: Alveolar cell death in hyperoxia-induced lung injury. Ann N Y Acad Sci 1010: 405-416, 2003.

8. Cavalcante GC, Schaan AP, Cabral GF, Santana-da-Silva MN, Pinto P, Vidal AF and Ribeiro-Dos-Santos A: A cell's fate: An overview of the molecular biology and genetics of apoptosis. Int J Mol Sci 20: 4133, 2019.

9. Sayers TJ: Targeting the extrinsic apoptosis signaling pathway for cancer therapy. Cancer Immunol Immunother 60: 1173-1180, 2011.

10. Marciniak SJ: Endoplasmic reticulum stress in lung disease. Eur Respir Rev 26: 170018, 2017.

11. Wei J, Rahman S, Ayaub EA, Dickhout JG and Ask K: Protein misfolding and endoplasmic reticulum stress in chronic lung disease. Chest 143: 1098-1105, 2013.

12. Wang J, Zhang Y, Liu X, Wang J, Li B, Liu Y and Wang J: Alantolactone enhances gemcitabine sensitivity of lung cancer cells through the reactive oxygen species-mediated endoplasmic reticulum stress and Akt/GSK3 $\beta$ pathway. Int J Mol Med 44: 1026-1038, 2019. 
13. Naiel S, Tat V, Padwal M, Vierhout M, Mekhael O, Yousof T, Ayoub A, Abed S, Dvorkin-Gheva A and Ask K: Protein misfolding and endoplasmic reticulum stress in chronic lung disease: Will cell-specific targeting be the key to the cure? Chest 157: 1207-1220, 2020.

14. Lu HY, Zhang J, Wang QX, Tang W and Zhang LJ: Activation of the endoplasmic reticulum stress pathway involving CHOP in the lungs of rats with hyperoxia-induced bronchopulmonary dysplasia. Mol Med Rep 12: 4494-4500, 2015.

15. Li M, Pan B, Shi Y, Fu J and Xue X: Increased expression of CHOP and LC3B in newborn rats with bronchopulmonary dysplasia. Int J Mol Med 42: 1653-1665, 2018.

16. Iurlaro R and Muñoz-Pinedo C: Cell death induced by endoplasmic reticulum stress. FEBS J 283: 2640-2652, 2016

17. Gong J, Wang XZ, Wang T, Chen JJ, Xie XY, Hu H, Yu F, Liu HL, Jiang XY and Fan HD: Molecular signal networks and regulating mechanisms of the unfolded protein response. J Zhejiang Univ Sci B 18: 1-14, 2017.

18. Jin C, Jin Z, Chen NZ, Lu M, Liu CB, Hu WL and Zheng CG: Activation of IRE1 $\alpha$-XBP1 pathway induces cell proliferation and invasion in colorectal carcinoma. Biochem Biophys Res Commun 470: 75-81, 2016.

19. Sha H, He Y, Chen H, Wang C, Zenno A, Shi H, Yang X, Zhang X and Qi L: The IRE1alpha-XBP1 pathway of the unfolded protein response is required for adipogenesis. Cell Metab 9: 556-564, 2009.

20. Jiang D, Niwa M and Koong AC: Targeting the IRE1 $\alpha-X B P$ branch of the unfolded protein response in human diseases. Semin Cancer Biol 33: 48-56, 2015.

21. Wu J, He GT, Zhang WJ, Xu J and Huang QB: IRE1 $\alpha$ signaling pathways involved in mammalian cell fate determination. Cell Physiol Biochem 38: 847-858, 2016.

22. Hou A, Fu J, Yang H, Zhu Y, Pan Y, Xu S and Xue X: Hyperoxia stimulates the transdifferentiation of type II alveolar epithelial cells in newborn rats. Am J Physiol Lung Cell Mol Physiol 308: L861-L872, 2015.

23. Cooney T and Thurlbeck W: The radial alveolar count method of Emery and Mithal: A reappraisal 1-postnatal lung growth Thorax 37: 572-579, 1982.

24. Herring MJ, Putney LF, Wyatt G, Finkbeiner WE and Hyde DM: Growth of alveoli during postnatal development in humans based on stereological estimation. Am J Physiol Lung Cell Mol Physiol 307: L338-L344, 2014.

25. Livak KJ and Schmittgen TD: Analysis of relative gene expression data using real-time quantitative PCR and the 2(-Delta Delta C(T)) method. Methods 25: 402-408, 2001

26. Wang M, Ye R, Barron E, Baumeister P, Mao C, Luo S, Fu Y, Luo B, Dubeau L, Hinton DR and Lee AS: Essential role of the unfolded protein response regulator GRP78/BiP in protection from neuronal apoptosis. Cell Death Differ 17: 488-498, 2010.

27. Zhang Y, Liu R, Ni M, Gill P and Lee AS: Cell surface relocalization of the endoplasmic reticulum chaperone and unfolded protein response regulator GRP78/BiP. J Biol Chem 285: $15065-15075,2010$.

28. Teng R, Jing $\mathrm{X}$, Michalkiewicz T, Afolayan A, Wu T and Konduri G: Attenuation of endoplasmic reticulum stress by caffeine ameliorates hyperoxia-induced lung injury. Am J Physiol Lung Cell Mol Physiol 312: L586-L598, 2017.
29. Chen Y and Brandizzi F: IRE1: ER stress sensor and cell fate executor. Trends Cell Biol 23: 547-555, 2013.

30. Adams CJ, Kopp MC, Larburu N, Nowak PR and Ali MMU: Structure and molecular mechanism of ER stress signaling by the unfolded protein response signal activator IRE1. Front Mol Biosci 6: 11, 2019.

31. Hetz C, Martinon F, Rodriguez D and Glimcher LH: The unfolded protein response: Integrating stress signals through the stress sensor IRE1 $\alpha$. Physiol Rev 91: 1219-1243, 2011.

32. Verma $G$ and Datta M: The critical role of JNK in the ER-mitochondrial crosstalk during apoptotic cell death. J Cell Physiol 227: 1791-1795, 2012.

33. Akiyama M, Liew CW, Lu S, Hu J, Martinez R, Hambro B, Kennedy RT and Kulkarni RN: X-box binding protein 1 is essential for insulin regulation of pancreatic $\alpha$-cell function. Diabetes 62: 2439-2449, 2013.

34. Casas-Tinto S, Zhang Y, Sanchez-Garcia J, Gomez-Velazquez M, Rincon-Limas DE and Fernandez-Funez P: The ER stress factor XBP1s prevents amyloid-beta neurotoxicity. Hum Mol Genet 20: 2144-2160, 2011

35. Tashiro E: Screening and identification of inhibitors of endoplasmic reticulum stress-induced activation of the IRE1 $\alpha-X B P 1$ branch. J Antibiot (Tokyo) 72: 899-905, 2019.

36. Chen L, Li Q, She T, Li H, Yue Y, Gao S, Yan T, Liu S, Ma J and Wang Y: IRE1 $\alpha$-XBP1 signaling pathway, a potential therapeutic target in multiple myeloma. Leuk Res 49: 7-12, 2016.

37. Hasegawa D, Calvo V, Avivar-Valderas A, Lade A, Chou HI, Lee YA, Farias EF, Aguirre-Ghiso JA and Friedman SL: Epithelial Xbp1 is required for cellular proliferation and differentiation during mammary gland development. Mol Cell Biol 35: $1543-1556,2015$.

38. Mehan S, Meena H, Sharma D and Sankhla R: JNK: A stress-activated protein kinase therapeutic strategies and involvement in Alzheimer's and various neurodegenerative abnormalities. J Mol Neurosci 43: 376-390, 2011.

39. Seki E, Brenner D and Karin M: A liver full of JNK: Signaling in regulation of cell function and disease pathogenesis, and clinical approaches. Gastroenterology 143: 307-320, 2012.

40. Feng J, Lu S, Ou B, Liu Q, Dai J, Ji C, Zhou H, Huang H and Ma Y: The role of JNk signaling pathway in obesity-driven insulin resistance. Diabetes Metab Syndr Obes 13: 1399-1406, 2020.

41. Wu Q, Wu W, Jacevic V, Franca TCC, Wang X and Kuca K: Selective inhibitors for JNK signalling: A potential targeted therapy in cancer. J Enzyme Inhib Med Chem 35: 574-583, 2020.

42. De Paepe ME, Gundavarapu S, Tantravahi U, Pepperell JR, Haley SA, Luks FI and Mao Q: Fas-ligand-induced apoptosis of respiratory epithelial cells causes disruption of postcanalicular alveolar development. Am J Pathol 173: 42-56, 2008.

This work is licensed under a Creative Commons Attribution-NonCommercial-NoDerivatives 4.0 International (CC BY-NC-ND 4.0) License. 